\title{
CXCR4-transfected human umbilical cord blood-derived mesenchymal stem cells exhibit enhanced migratory capacity toward gliomas
}

\author{
SOON A. PARK ${ }^{1}$, CHUNG HEON RYU ${ }^{1}$, SEONG MUK KIM ${ }^{1}$, JUNG YEON LIM ${ }^{1}$, SANG IN PARK ${ }^{1}$, \\ CHANG HYUN JEONG ${ }^{1}$, JIN AE JUN ${ }^{1}$, JI HYEON OH ${ }^{1}$, SUN HWA PARK ${ }^{1}$, WONIL OH ${ }^{3}$ and SIN-SOO JEUN ${ }^{1,2}$ \\ Departments of ${ }^{1}$ Biomedical Science, College of Medicine, and ${ }^{2}$ Neurosurgery, Seoul St. Mary's Hospital, The Catholic \\ University of Korea; ${ }^{3}$ Medipost Biomedical Research Institute, Medipost Co., Ltd., Seoul, Republic of Korea
}

Received July 30, 2010; Accepted September 2, 2010

DOI: 10.3892/ijo_00000828

\begin{abstract}
Mesenchymal stem cells (MSCs) can be used as a delivery vehicle for gene therapy against brain tumors, because these cells have a migratory capacity toward glioma cells. Soluble factors including chemokines or growth factors expressed and released by glioma cells mediate the tropism of MSCs for gliomas. Among them, stromal cell-derived factor- $1 \alpha(\mathrm{SDF}-1 \alpha)$ has been identified as a key molecule related to the tropism of MSC in many cancers containing gliomas. In this study, we found that overexpression of the SDF-1 $\alpha$ receptor, CXCR4, on human umbilical cord bloodderived MSCs (hUCB-MSCs) enhanced the migratory capacity of MSCs toward gliomas. We showed that hUCB-MSCs have the migration ability toward the glioma cell lines and primary glioma cells. SDF-1 $\alpha$ treatment increased the migration capacity of hUCB-MSCs in a dose-dependent manner and inhibition of SDF- $1 \alpha$ or CXCR 4 by treatment with the anti-SDF-1 $\alpha$ or the CXCR4 antagonist AMD3100 blocked the migration capacity of hUCB-MSCs toward glioma cells. Furthermore, CXCR4-overexpressed hUCB-MSCs (hMSCsCXCR4) showed a stronger migration capacity toward glioma cells in vitro compared with control MSCs, and also exhibited enhanced migration to glioma cells in an intracranial human malignant glioma xenograft model. These results indicate that SDF-1 $\alpha /$ CXCR 4 could be involved in recruitment of hUCB-MSCs to glioma cells and that overexpression of CXCR4 may be a useful tool for stem cell-based glioma therapy.
\end{abstract}

Correspondence to: Dr Sin-Soo Jeun, Department of Neurosurgery, Seoul St. Mary's Hospital, The Catholic University of Korea, 505 Banpo-dong Seocho-ku, Seoul 137-701, Republic of Korea E-mail: ssjeun@catholic.ac.kr

Key words: umbilical cord blood-derived mesenchymal stem cell, glioma, migration, SDF-1 $\alpha, \mathrm{CXCR} 4$

\section{Introduction}

Malignant gliomas are a type of tumor that arises from glial cells in the brain. The prognosis of patients with median survival is still 1 year because gliomas are refractory to conventional treatments, such as extensive surgical resection, radiation, chemotherapy and gene therapy $(1,2)$. This treatment resistance is partly due to the infiltration and invasion of gliomas into surrounding brain architecture. Recent new therapies for brain tumors have prompted the proposal, that neural stem cells (NSCs) with tumor tropism may be used as a vehicle for the delivery of therapeutic agents to target the tumor (3-5).

However, the clinical application of NSCs is limited by ethical and logistic problems related to their isolation and immunologic incompatibility in allogenic transplantation $(6,7)$. Ideally stem cells should be autologous cells that can be harvested without difficulty from the patient. However, the number and differentiation ability of bone marrow-derived mesenchymal stem cells (BM-MSCs) significantly decreases with age (8). Human umbilical cord blood (hUCB) is an alternative source of MSCs, with several studies showing that hUCB-derived MSCs (hUCB-MSCs) are similar to $\mathrm{BM}-\mathrm{MSC}$ regarding cell characteristics and multilineage differentiation potential $(9,10)$. Additionally, we have previously shown that hUCB-MSCs have the migratory capacity and tropism for gliomas, and can be used as a therapeutic vehicle for glioma therapy $(11,12)$. However, the mechanism underlying the glioma tropism of hUCB-MSCs is not well understood.

Directed cell migration is initiated in response to chemoattractants that include, cytokines and growth factors. Therefore, it is essential to understand the various factors that regulate hUCB-MSC migration to gliomas. Tumors and the surrounding inflamed tissue are known to express interleukin (IL)-1 $\alpha$, IL-1ß, transforming growth factor (TGF) $\beta$, IL-10, tumor necrosis factor (TNF) $\alpha, \mathrm{TNF} \beta$, interferon-gamma (IFN $\gamma$ ), and monocyte chemoattractant protein-1 (MCP-1) $(13,14)$, and stem cells express the corresponding receptors $(15,16)$. Thus, factors and their receptors might play roles in 
the specific migration of MSCs towards infarcts and brain tumors. In our previous study, we demonstrated that hUCBMSCs have a strong migration capacity toward glioma secreted IL-8 (11). Other factors expressed by tumors, including stromal cell-derived factor- $1 \alpha(\mathrm{SDF}-1 \alpha)$, vascular endothelial growth factor (VEGF), urokinase plasminogen activator (UPA), epidermal growth factor (EGF), and transmembrain protein 18 (TMEM18) have also been shown to be involved in migration of MSCs migration (17-22).

SDF- $1 \alpha$ is a member of the CXC chemokine family, and its receptor CXCR4 was originally described as a regulator of cell interactions in the immune system. SDF-1 $\alpha /$ CXCR4 in the cental nervous system (CNS) suggests an important function in the migration of stem cells during CNS development. Recently, an essential role of SDF- $1 \alpha / \mathrm{CXCR} 4$ in tumor metastasis and vascularization was reported (23). Moreover, SDF- $1 \alpha / C X C R 4$ plays an important and unique role in the regulation of stem cell trafficking. Its chemokine regulates the migration of hematopoietic stem cells (HSCs), their homing/migration in major bone marrow, and accumulation of immune cells in inflammatory tissue. Additionally, proper functioning of the SDF- $1 \alpha /$ CXCR 4 axis is important in engraftment, proliferation, and survival of HSCs (24). Therefore, we hypothesize that SDF-1/CXCR4 signaling plays an important role in tumor tropism of hUCB-MSCs and examined whether overexpression of CXCR4 on hUCB-MSCs enhances MSCs migratory capacity toward gliomas.

\section{Materials and methods}

Preparation of hUCB-MSC. Human UCB samples were obtained with consent of the mothers. The hUCB-MSCs were separated and maintained as previously described (9). hUCBMSCs were cultured in $\alpha$-MEM (Invitrogen) media containing $10 \%$ fetal bovine serum (FBS).

Cancer cell line and primary cell culture. U-87MG and U251MG cells were obtained from the American Type Culture Collection and were maintained in Eagle's MEM (EMEM; Hyclone) and DMEM (Hyclone), respectively. Normal human astrocytes were obtained from the Applied Cell Biology Research Institute and were cultured in DMEM. After ethical approval and written informed consent, fresh specimens of human brain tumors were harvested from patients undergoing surgery. Briefly, tissues were washed, finely minced, and then separated into brain tissue fractions using differential centrifugation. Each cell population was then cultured in DMEM. All media were supplemented with $2 \mu \mathrm{mol} / 1 \mathrm{~L}-$ glutamine, $100 \mathrm{U} / \mathrm{ml}$ penicillin, $100 \mu \mathrm{g} / \mathrm{ml}$ streptomycin, and $10 \%$ FBS purchased from Hyclone. Cells were incubated at $37^{\circ} \mathrm{C}$ in a humidified atmosphere containing $5 \% \mathrm{CO}_{2}$.

Reverse transcription PCR. Total RNA was prepared from glioma cell lines and primary glioma cells using TRIzol (Invitrogen) according to the manufacturer's instructions. cDNA was synthesized using $3 \mu \mathrm{g}$ total RNA and oligo(dT) primer and Superscript II polymerase for reverse transcription PCR (Invitrogen). PCR amplifications consisted of a total of 35 cycles of denaturation at $94^{\circ} \mathrm{C}$ for $30 \mathrm{sec}$, annealing at $52^{\circ} \mathrm{C}$ for $30 \mathrm{sec}$, extension at $72^{\circ} \mathrm{C}$ for 1 min with a first denaturation at $94^{\circ} \mathrm{C}$ for $10 \mathrm{~min}$, and final extension at $72^{\circ} \mathrm{C}$ for $10 \mathrm{~min}$. Target primers sequences used were as follows: SDF-1 $\alpha$ forward: 5'-ATGAACGCCAAGGTCGTGGTC-3', SDF-1 $\alpha$ reverse: 5'-ACCTCTTGTTTAAAGCTTTC-3' and GAPDH forward: 5'-TCCATGACAACTTTGGTATCG-3', GAPDH reverse: 5'-TGTAGCCAAATTCGTTGTCA-3'.

Transfection of hUCB-MSCs with CXCR4. Transfections of the hUCB-MSCs were conducted using the pcDNA-CXCR4 plasmid and the control of the moc-vector. Cells were resuspended in Resuspension buffer ${ }^{\circledR}$ (Invitrogen) at a density of $1 \times 10^{6}$ cells $/ \mathrm{ml}$ and incubated with $2 \mu \mathrm{g}$ of plasmid. The Neon transfection system (Invitrogen) was performed at room temperature using program 3 (1600 voltage, $20 \mathrm{~ms}$, one pulse). After transfection, cells were distributed into $35-\mathrm{mm}$-diameter cell culture dishes and placed at $37^{\circ} \mathrm{C}$ in a $5 \% \mathrm{CO}_{2}$-humidified atmosphere.

For detection of transfected cells was used flow cytometric analysis. hUCB-MSCs were analyzed for the surface expression of SDF-1 receptor with anti-human CXCR4. Briefly, 2x $10^{5}$ cells were incubated with anti-CD 184 primary antibody (rat polyclonal anti-human CXCR4) for $20 \mathrm{~min}$ at $4^{\circ} \mathrm{C}$, and then washed three times with PBS. Following the final wash, the cells were resuspended in PBS and incubated with Alexa fluor 488 goat anti-rat secondary antibody (Invitrogen) for $20 \mathrm{~min}$ at $4^{\circ} \mathrm{C}$. After washing with PBS, the expressions of these receptors were analyzed by flow cytometry using the FACS Vantage SE system (BectonDickinson).

In vitro migration assay. Migration of human hUCB-MSCs was determined using Transwell plates (Corning Costar) that were $6.5 \mathrm{~mm}$ in diameter with $8-\mu \mathrm{m}$ pore size filters. hUCBMSCs $\left(3 \times 10^{4}\right.$ cells) were added to the upper chamber, and $600 \mu 1$ of conditioned medium or SDF- $1 \alpha$ was added to the bottom chamber. For the experiment of migration of CXCR 4 overexpressed hUCB-MSCs, conditioned medium with $1 / 3$ diluted were used. After incubation for $5 \mathrm{~h}$ at $37^{\circ} \mathrm{C}$ in an atmosphere of $5 \% \mathrm{CO}_{2}$, cells that had not migrated from the upper side of the filters were removed with a cotton swab and the lower side was stained using a Diff-Quik ${ }^{\mathrm{TM}}$ three-step stain set (Sysmex), mounted on glass slides. At least five random fields of view on each membrane were selected, and cells were counted using light microscopy with an Axiovert 200 microscope (Carl Zeiss) at magnification x200. Each experiment was performed in duplicate, with results expressed as mean \pm standard deviation (SD).

In vivo migration assay. Human U-251MG glioma cells ( $5 \times 10^{4}$ cells in $3 \mu 1 \mathrm{PBS}$ ) were injected into the right striatum of the nude mouse brain (AP $+1.0 \mathrm{~mm}, \mathrm{ML}+2.0 \mathrm{~mm}$, and DV - $3.0 \mathrm{~mm}$ from bregma and dura) using a 10 AL Hamilton syringe connected with a 30 -gauge needle at a speed of 0.5 $\mu 1 / \mathrm{min}$. Then, $10^{5}$ cells of red fluorescent PKH26 dye (SigmaAldrich)-labeled CXCR4-overexpressing hMSCs were mixed with an equal number of green fluorescent PKH67 dye (SigmaAldrich)-labeled vector control-hMSCs and injected into the contralateral side of the mouse brain. The brain samples were collected 10 days later for cryosectioning (16- $\mu \mathrm{m}$-thick sections) and counter staining. Migrating cells were detected 
A
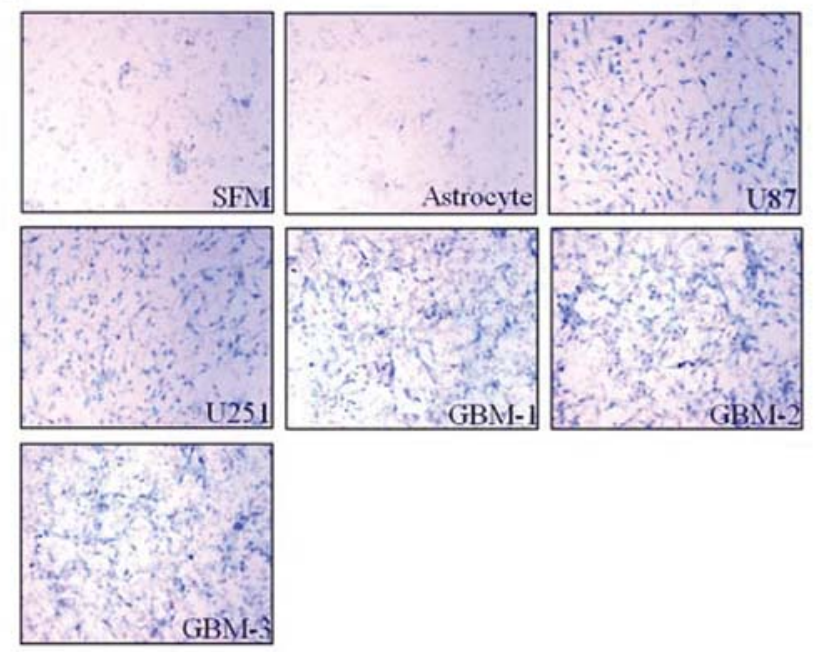

B

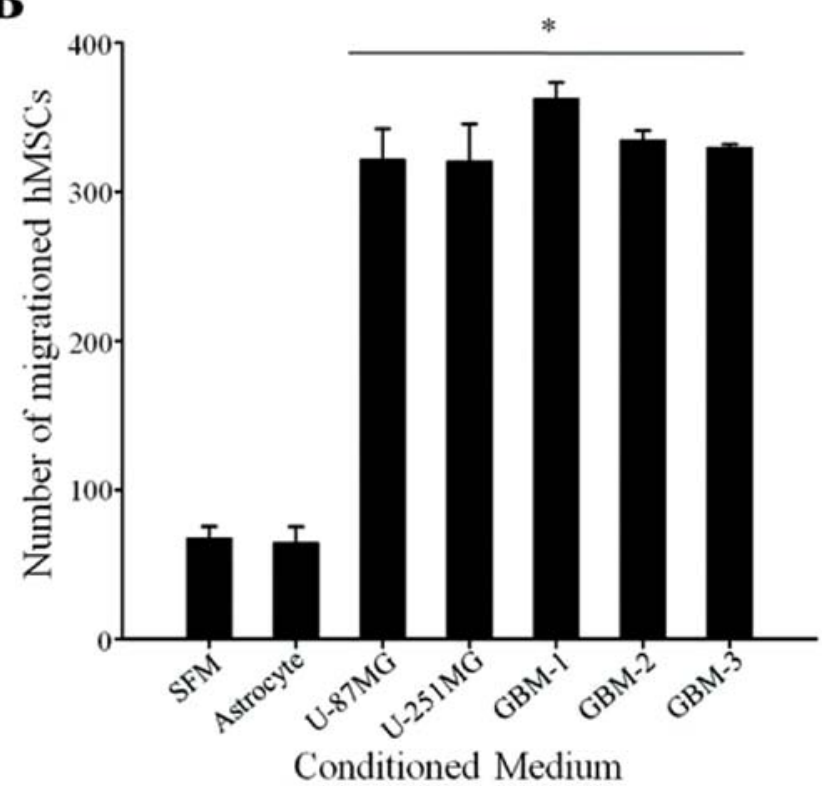

Figure 1. In vitro migration assay of hUCB-MSCs. (A) Representative photomicrographs of stained filters showing migrating hUCB-MSCs (magnification $\mathrm{x} 100$ ). (B) hUCB-MSCs showed a strong migration to conditioned medium derived from glioma cell lines and primary brain cancer cells. Cell migration was compared and evaluated after staining by photographing randomly selected fields and enumerating migrating cells. Serum-free medium (SFM) and astrocytes were used as a negative control. All conditions were repeated in duplicate, with five random fields of view counted on each membrane. Bars, mean; error bars, standard error; $\mathrm{p}<0.05$.

using an AX70 fluorescence microscope (Olympus) or an LSM 510 Meta confocal microscope (Carl Zeiss).

Statistical analysis. Data from migration assays were compared by Student's t-test for two samples with unequal variances. Statistical significance was determined at the $>0.05$ level.

\section{Results}

Migration of hUCB-MSCs toward gliomas. Stem cells exhibit extensive tropism for experimental gliomas (25). To test this
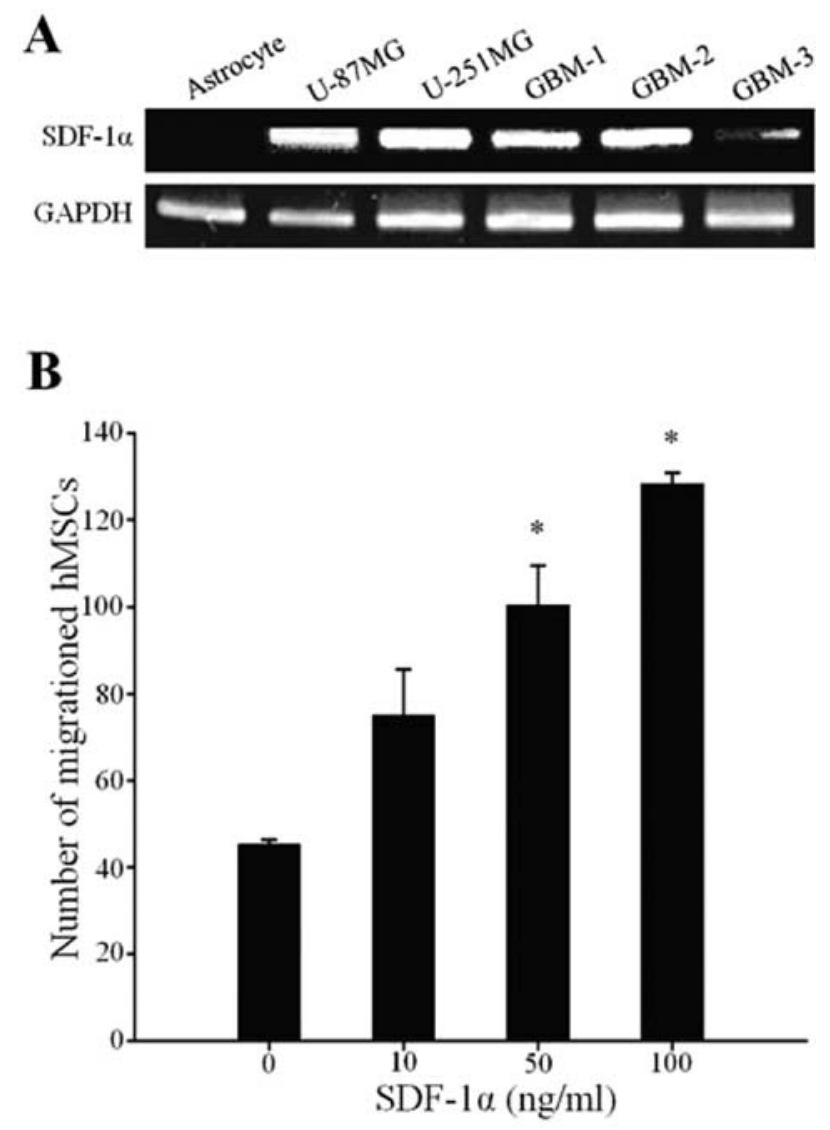

Figure 2. In vitro migratory responses of hUCB-MSCs and the role of the SDF-1 $\alpha /$ CXCR4 pathways. (A) The expression of SDF-1 $\alpha$ in glioma cell lines and primary glioma cells by RT-PCR. (B) Graph showing migration of hUCB-MSCs in response to recombinant human SDF-1 $\alpha(0-100 \mathrm{ng} / \mathrm{ml})$. Migration of hUCB-MSCs increased in an SDF-1 $\alpha$ dose-dependent manner. Bars, mean; error bars, standard error; $\mathrm{p}<0.05$.

in hUCB-MSCs, in vitro migration assays using Transwell plates were conducted. hUCB-MSCs were cultured in the upper chamber and the target glioma cell (U-87MG, U-251MG, or primary cells)-conditioned medium was placed in the lower chamber. Conditioned medium from normal human astrocytes was used as the negative control. Only a few cells migrated toward serum-free medium and conditioned medium from normal human astrocytes, whereas the migration of hUCB-MSCs was significantly stimulated by human glioma cell lines or human glioma primary cells-conditioned medium (Fig. 1). These results were consistent with the capability of human glioma cells of stimulating the migration of hUCBMSCs.

Glioma-secreted SDF-1a induces CXCR4-dependent migration of $h U C B-M S C s$. Factor released from glioma cells may be a potential chemoattractant involved in the tropism of stem cells (25). Among the various factors, SDF- $1 \alpha$ has a potential role in stem cell tropism toward glioma. For example, SDF- $1 \alpha$ from gliomas regulates the migration of neural stem cells (26). We confirmed expression of SDF-1 $\alpha$ by RT-PCR in glioma cells. As the result, SDF-1 $\alpha$ was expressed in all glioma cell lines and glioma primary cells (Fig. 2A). To examine the involvement of SDF- $1 \alpha$ in the migration of hUCB-MSCs, hUCB-MSCs were treated with recombinant SDF-1 $\alpha$. The 

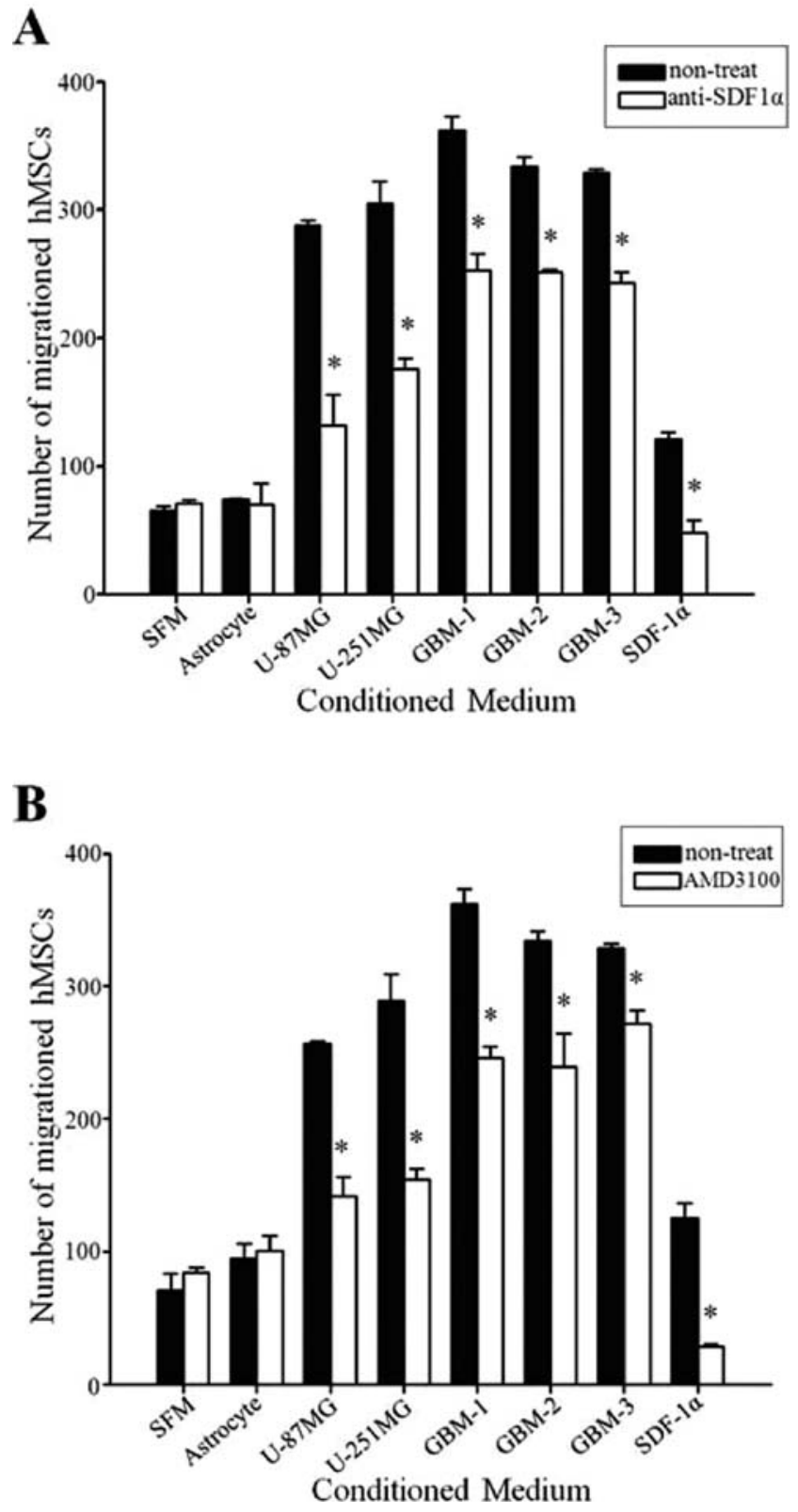

Figure 3. Glioma-secreted SDF-1 $\alpha$ induces hUCB-MSCs migration. (A) Migration of MSCs when challenged with glioma conditioned medium before and after addition of SDF-1 $\alpha$ antibody. (B) Addition of the CXCR4 antagonist AMD3100 significantly decreased hUCB-MSCs migration toward glioma-conditioned medium compared to untreated hUCB-MSCs. Bars, mean; error bars, standard error; $\mathrm{p}<0.05$.

migration of hUCB-MSCs was significantly stimulated by SDF- $1 \alpha$ in a dose-dependent manner (Fig. 2B), consistent with the suggestion. The SDF- $1 \alpha$ affects the migration of hUCB-MSCs.

To more precisely define the involvement of SDF- $1 \alpha$ in MSCs migration, glioma cell SDF- $1 \alpha$ was neutralized by incubation in the presence of an antibody to SDF- $1 \alpha$ in conditioned medium from glioma cells. Many cells migrated toward the glioma cell-conditioned medium incubated with an isotype-matched nonspecific antibody, but migration of hUCB-MSCs decreased significantly in the anti-SDF-1 $\alpha$ treated glioma cell-conditioned medium (Fig. 3A). In addition, hUCB-MSCs migration markedly decreased in the glioma
A

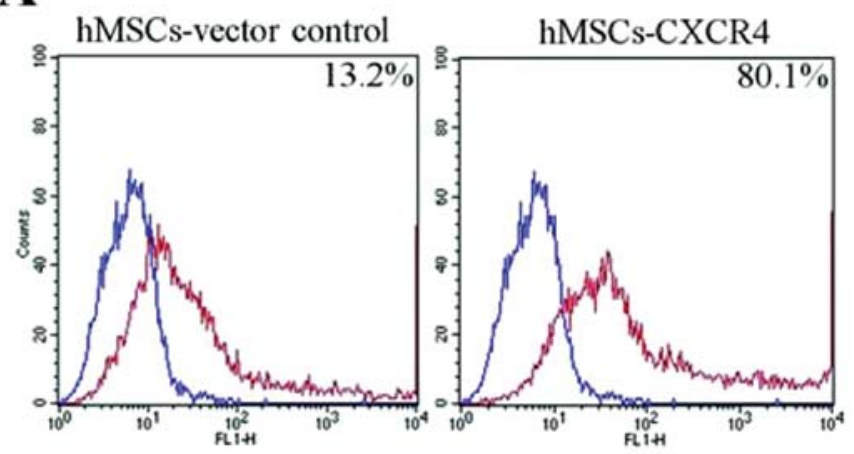

B

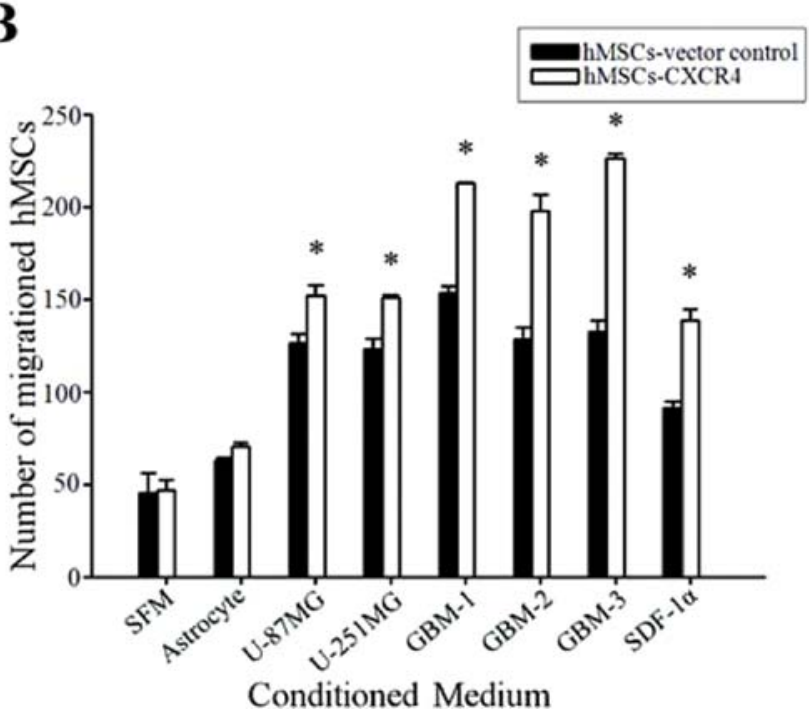

Figure 4. Effect of CXCR4 overexpression on migration of hUCB-MSCs. (A) FACS confirmation of CXCR4 expression. hMSCs-vector control (left) and hMSCs-CXCR4 (right) were stained with anti-human CXCR4 (red lines), or appropriate isotype-matched controls (blue lines). The cells were then labeled with FITC. (B) CXCR4 overexpression increased the migration activity of hUCB-MSCs in the Transwell system. Bars, mean; error bars, standard error; $\mathrm{p}<0.05$.

cell-conditioned medium incubated with the SDF-1 $\alpha$ receptor CXCR4 antagonist AMD3100 (Fig. 3B). These results were consistent with the view that SDF-1 $\alpha$ induces hUCB-MSCs migration toward glioma cells via the CXCR4 receptor.

Overexpression of $C X C R 4$ enhances the glioma-specific migration ability of UCB-MSCs. Previous results showed that SDF-1 $\alpha$ induced hUCB-MSCs migration for glioma cells via its receptor CXCR4 (27). Presently, the endogenous expression of CXCR4 in hUCB-MSCs was examined using fluorescence-activated cell sorting (FACS). The percentage of CXCR4 positive cells was only $13 \%$. To enhance hUCB-MSCs tropism for glioma cells, cells were transfected with a CXCR4 expression plasmid using a neon transfection system, by electrical gene transfer. The percentage of CXCR4 -positive cell was $80 \%$ after transfection (Fig. 4A).

The next experiment investigated the migration activity of CXCR4-overexpressed hUCB-MSCs (hMSCs-CXCR4) toward glioma-conditioned medium and SDF-1 $\alpha$. hMSCsCXCR4 displayed significantly higher migration capacity 

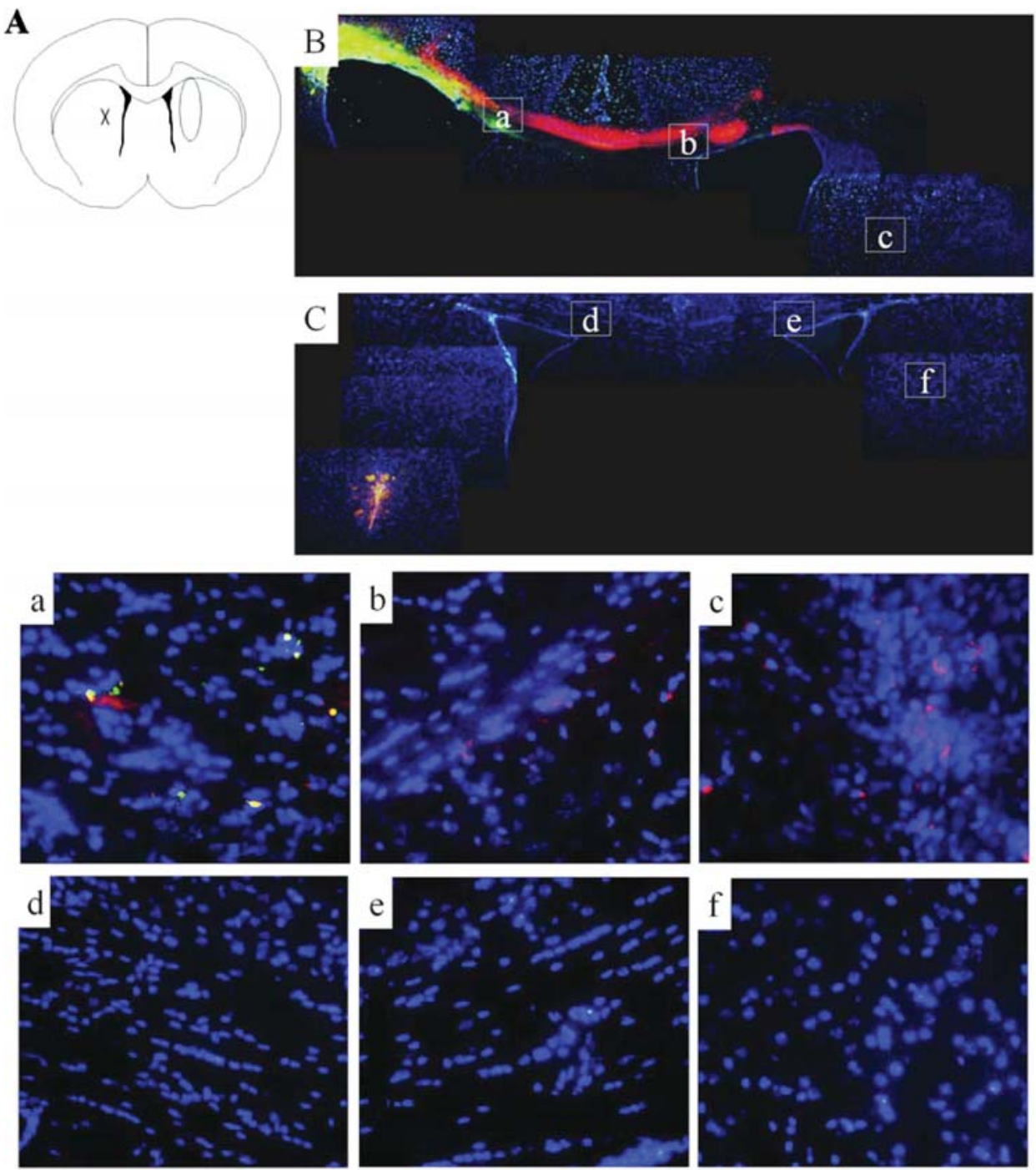

Figure 5. CXCR4 overexpression increases migration of UCB-MSCs toward human glioma cells in the nude mouse brain. PKH26-labeled hMSCs-CXCR4 and PKH67-labeled hMSCs-vector control were mixed together and injected into the left side of the mouse brain contralateral to a U-251MG glioma inoculation site (right). Ten days later, the brains were sectioned for fluorescence microscopy assessment of cell migration. (A) Schematic of tumor site on the right and mixing cells transplantation site on the left. (B) Mixing cells transplanted into the normal mouse brain (magnification x200). (a-c) A higher magnification view (x400) of the white box denoted in (B). (C) The migration of mixed cells migration away from the contalateral site of gliomas (magnification x200). (d-f) A higher magnification view (x400) of the white box denoted in (C).

when compared with the control hUCB-MSCs. But, migration of hMSCs-CXCR4 and the control hMSCs to serum-free medium remained similar (Fig. 4B).

To assess the in vivo migration capacity of hMSC-CXCR4, PKH-26-labeled hMSCs-CXCR4 were injected together with PKH-67-labeled control hUCB-MSCs into the contralateral hemisphere of tumors in a U-251MG glioma model. PKH-26labeled cells migrated more extensively from the initial injection site toward the tumor mass along the corpus callous 10 days after injection of the cells (Fig. 5B and a-c). These observations were consistent with the view that hMSCsCXCR4 possess excellent migratory capacity and glioma tropism in vitro.

\section{Discussion}

Despite advances in surgical and adjuvant therapy, the prognosis for malignant gliomas remains dismal. Viral vector-mediated therapeutic gene delivery involving direct injection into the primary brain tumor or postoperative tumor cavity often fails to reach outgrowing tumor islands because of the migratory abilities of the tumor cells and their marked infiltration into normal brain (28). Therefore, current treatments that rely on targeting the tumor mass are often ineffective.

Recently, the use of MSCs as vehicles for delivering therapeutic genes of glioma treatment was described (25). Cell-based gene therapy for gliomas depends crucially on tumor tropism. Previously, we reported that hUCB-MSCs can migrate toward human glioma cell lines $(11,12)$. In addition, it was presently observed that hUCB-MSCs seem to have a migratory capacity toward conditioned medium from primary glioma cells (Fig. 1). However, to date, only a very small proportion of surviving grafted cells have been shown to be capable of integrating into the inflammatory sites (29). In addition, with increased understanding of the mechanistic underpinnings of MSC migration, the potential for enhancing 
MSC-targeted therapies appears promising (30). Therefore, our ultimate goal is to increase the specificity of hUCB-MSCs migration to gliomas.

In recent studies, soluble factors secreted from tumors were suggested as a critical factor for stem cell attraction. Glioma and the surrounding inflamed tissue express migratory factors, MCP-1, IL-8, uPA, EGF, and VEGF (20,31-34). Among the various factors, we focused on SDF- $1 \alpha$ and its receptor CXCR4, which is known to mediate bone marrow-derived cell trafficking under normal and pathologic conditions (35). It has been reported that high-grade gliomas secrete significant levels of SDF-1 $\alpha$, and that the expression of this receptor CXCR4 correlates with the histological grade and invasive capacity of gliomas, as well as with tumor cell survival (36).

Ehtesham et al showed that SDF-1 $1 /$ CXCR4 is important for glioma tropism of fetal neural progenitor/stem cells (26). The present data demonstrate that the functionality of the CXCR4, as judged by the chemotactic response of hUCB-MSCs to an SDF-1 $\alpha$ gradient (Fig. 2). Presence of anti-SDF- $1 \alpha$ or the CXCR4 antagonist AMD 3100 significantly inhibited chemotaxis of hUCB-MSCs toward glioma cell-conditioned medium, supporting a key role of SDF-1 $\alpha /$ CXCR4 in hUCB-MSCs including gliomas (Fig. 3).

Non-viral methods of transfection are receiving increasing attention, given the several potential advantages over recombinant viruses. The present study used electrical mediation (the Neon transfection system) to transfect CXCR4 into hUCB-MSCs to examined the effect of CXCR4 on MSCs migration toward gliomas. The Neon transfection system is a new electroporation technology that uses a pipette tip as an electroporation space and a gold-coated electrode surface. Wang et al reported it to be safe and efficient in the transduction of human adipo-derived stem cells (hADSCs) (37), and presently allowed the successful overexpression of CXCR4 in hUCB-MSCs. Strong hMSCs-CXCR4 migration toward glioma cells was evident as compared with hUCB-MSCs (Fig. 4).

In vivo assessment of $\mathrm{PKH} 26$-labeled cells demonstrated that hMSCs-CXCR4 strongly migrate toward distant intracranial tumors, in contrast to the weak migration of hUCBMSCs (Fig. 5). These observations are consistent with the view that hMSCs-CXCR4 that infiltrate a tumor may be a powerful vehicle to deliver suicide genes, proapoptotic genes, or immune modulators to the tumor. Recently, Sato et al demonstrated that EGFR modified bone marrow stromal cells and also transfected of INF- $\alpha$ into EGFR-MSCs strengthens this therapy approaches for refractory brain tumors (21).

In conclusion, the present data indicate that SDF-1/ CXCR4 signaling is important for governing the process of hUCB-MSCs migration toward glioma cells. CXCR4 overexpression enhances migration of MSCs toward gliomas. Therefore, it is likely that hMSCs-CXCR4 will have their greatest applicability as a vehicle for delivering therapeutic genes for the tumor treatment.

\section{Acknowledgments}

This study was supported by a grant form the National R\&D Program for Cancer Control (0820040) and the Korea Healthcare Technology R\&D Project (A092258), Ministry of Health, Welfare and Family Affairs, Republic of Korea.

\section{References}

1. Davis FG, McCarthy BJ and Berger MS: Centralized databases available for describing primary brain tumor incidence, survival, and treatment: Central Brain Tumor Registry of the United States; Surveillance, Epidemiology, and End Results; and National Cancer Data Base. J Neurooncol 1: 205-211, 1999.

2. De Angelis LM: Brain tumors. N Engl J Med 344: 114-123, 2001.

3. Aboody KS, Brown A, Rainov NG, et al: Neural stem cells display extensive tropism for pathology in adult br ain: evidence from intracranial gliomas. Proc Natl Acad Sci USA 97: 12846-12851, 2000

4. Ehtesham M, Kabos P, Kabosova A, Neuman T, Black KL and Yu JS: The use of interleukin 12-secreting neural stem cells for the treatment of intracranial glioma. Cancer Res 62: 5657-5663, 2002.

5. Yipa S, Sabetrasekh R, Sidmand RL and Snyder EY: Neural stem cells as novel cancer therapeutic vehicles. Eur J Cancer 42: 1298-1308, 2006.

6. Ehtesham M, Stevenson CB and Thompson RC: Stem cell therapies for malignant glioma. Neurosurg Focus 19: E5, 2005.

7. Nakamizo A, Marini F, Amano T, et al: Human bone marrowderived mesenchymal stem cells in the treatment of gliomas. Cancer Res 65: 3307-3318, 2005.

8. Tokalov SV, Grüner S, Schindler S, Iagunov AS, Baumann M and Abolmaali ND: A number of bone marrow mesenchymal stem cells but neither phenotype nor differentiation capacities changes with age of rats. Mol Cells 24: 255-260, 2007.

9. Yang SE, Ha CW, Jung MH, et al: Mesenchymal stem/progenitor cells developed in cultures from UC blood. Cytotherapy 6: 476-486, 2004.

10. Qiao C, Xu W, Zhu W, et al: Human mesenchymal stem cells isolated from the umbilical cord. Cell Biol Int 32: 8-15, 2008

11. Kim DS, Kim JH, Lee JK, et al: Overexpression of CXC chemokine receptors is required for the superior glioma-tracking property of umbilical cord blood-derived mesenchymal stem cells. Stem Cells Dev 18: 511-519, 2009.

12. Kim SM, Lim JY, Park SI, et al: Gene therapy using TRAILsecreting human umbilical cord blood-derived mesenchymal stem cells against intracranial glioma. Cancer Res 68: 9614-9623, 2008.

13. Yamanaka R, Tanaka R, Saitoh T and Okoshi S: Cytokine gene expression on glioma cell lines and specimens. J Neurooncol 21: 243-247, 1994.

14. Kielian T, Rooijen NV and Hickey WF: MCP-1 expression in CNS-1 astrocytoma cells: implications for macrophage infiltration into tumors in vivo. J Neurooncol 56: 1-12, 2002.

15. Chamberlain G, Wright K, Rot A, Ashton B and Middleton J: Murine mesenchymal stem cells exhibit a restricted repertoire of functional chemokine receptors: comparison with human. Plos One 3: e2934, 2008.

16. Ponte AL, Marais E, Gallay N, et al: The in vitro migration capacity of human bone marrow mesenchymal stem cells: comparison of chemokine and growth factor chemotactic activities. Stem Cells 25: 1737-1745, 2007.

17. Van der Meulen AA, Biber K, Lukovac S, Balasubramaniyan V, den Dunnen WF, Boddeke HW and Mooij JJ: The role of CXCL12-CXCR4 signalling in the migration of neural stem cells towards a brain tumour. Neuropathol Appl Neurobiol 35: 579-591, 2009

18. Xu Q, Yuan X, Xu M, et al: Chemokine CXC receptor 4mediated glioma tumor tracking by bone marrow-derived neural progenitor/stem cells. Mol Cancer Ther 9: 2746-2753, 2009.

19. Schmidt NO, Przylecki W, Yang W, et al: Brain tumor tropism of transplanted human neural stem cells is induced by vascular endothelial growth factor. Neoplasia 7: 623-629, 2005.

20. Gutova M, Najbauer J, Frank RT, et al: uPA and uPAR mediate human stem cell tropism to malignant solid tumors. Stem Cells 26: 1406-1413, 2008.

21. Sato H, Kuwashima N, Sakaida T, et al: Epidermal growth factor receptor-transfected bone marrow stromal cells exhibit enhanced migratory response and therapeutic potential against murine brain tumors. Cancer Gene Ther 12: 757-768, 2005.

22. Jurvansuu J, Zhao Y, Leung DSY, Boulaire J, Yu YH, Ahmed S and Wang S: Transmembrane protein 18 enhances the tropism of neural stem cells for glioma cells. Cancer Res 68: 4614-4622, 2008. 
23. Miller RJ, Banisadr G and Bhattacharyya BJ: CXCR4 signaling in the regulation of stem cell migration and development. J Neuroimmunol 198: 31-38, 2008.

24. Kahn J, Byk T, Jansson-Sjostrand L, et al: Overexpression of CXCR4 on human $\mathrm{CD} 34^{+}$progenitors increases their proliferation, migration, and NOD/SCID repopulation. Blood 103: 2942-2949, 2004.

25. Xu F and Zhu J-H: Stem cells tropism for malignant gliomas. Neurosci Bull 23: 363-369, 2007.

26. Ehtesham M, Yuan X, Kabos P, et al: Glioma tropic neural stem cells consist of astrocytic precursors and their migratory capacity is mediated by CXCR4. Neoplasia 6: 287-293, 2004.

27. Son BR, Marquez-Curtis LA, Kucia M, et al: Migration of bone marrow and cord blood mesenchymal stem cells in vitro is regulated by stromal-derived factor-1-CXCR4 and hepatocyte growth factor-c-met axes and involves matrix metalloproteinases. Stem Cells 24: 1254-1264, 2006.

28. Kramm CM, Sena-Esteves M, Barnett FH, et al: Gene therapy for brain tumors. Brain Pathol 5: 345-381, 1995.

29. Johnson TV, Bull ND and Martin KR: Identification of barriers to retinal engraftment of transplanted stem cells. Invest Ophthalmol Vis Sci 51: 960-970, 2010.

30. Spaeth E, Klopp A, Dembinski J, Andreeff M and Marini F Inflammation and tumor microenvironments: defining the migratory itinerary of mesenchymal stem cells. Gene Ther 15: $730-738,2008$
31. Magge SN, Malik SJ, Royo NC, et al: Role of monocyte chemoattractant protein-1 (MCP-1/CCL2) in migration of neural progenitor cells toward glial tumors. J Neurosci Res 87: 1547-1555, 2009.

32. Brat DJ, Bellail AC and van Meir EG: The role of interleukin-8 and its receptors in gliomagenesis and tumoral angiogenesis. Neurooncology 7: 122-133, 2005.

33. Dunn IF, Heese O and Black PM: Growth factors in glioma angiogenesis: FGFs, PDGF, EGF, and TGFs. J Neurooncol 50: 121-137, 2000.

34. Schichor C, Birnbaum T, Etminan N, et al: Vascular endothelial growth factor A contributes to glioma-induced migration of human marrow stromal cells (hMSC). Exp Neurol 199: 301-310, 2006

35. Imitola J, Raddassi K, Park KI, et al: Directed migration of neural stem cells to sites of CNS injury by the stromal cellderived factor $1 \alpha / \mathrm{CXC}$ chemokine receptor 4 pathway. Proc Natl Acad Sci USA 101: 18117-18122, 2004.

36. Rempel SA, Dudas S, Ge S and Gutiérrez JA: Identification and localization of the cytokine SDF1 and its receptor, CXC chemokine receptor 4 , to regions of necrosis and angiogenesis in human glioblastoma. Clin Cancer Res 6: 102-111, 2000.

37. Wang YH, Ho ML, Chang JK, Chu HC, Lai SC and Wang GJ: Microporation is a valuable transfection method for gene expression in human adipose tissue-derived stem cells. Mol Ther 17: 302-308, 2009. 